\title{
Long-Range Surface Plasmon Resonance Configuration for Enhancing SERS with an Adjustable Refractive Index Sample Buffer to Maintain the Symmetry Condition
}

\author{
Yu Liu, Haitao Zhang, Yijia Geng, Shuping Xu, Weiqing Xu, Jie Yu, Wenyuan Deng, Bo Yu, \\ and Liping Wang*
}

Cite This: ACS Omega 2020, 5, 32951-32958

Read Online

ABSTRACT: We propose a method to maintain the symmetry condition of the refractive index with respect to a dielectric buffer layer for a long-range surface plasmon resonance (LRSPR) configuration. The symmetry condition was maintained by changing the concentration of the ethylene glycol aqueous solution (sample buffer layer) to match the refractive index of the $\mathrm{MgF}_{2}$ film. Maintenance of the symmetry condition is necessary for exciting the LRSPR mode and increasing the electric field intensity near the film. We used a four-phase Kretschmann resonance setup composed of a $\mathrm{K} 9$ prism, $\mathrm{MgF}_{2}$ film, Ag film, and sample buffer

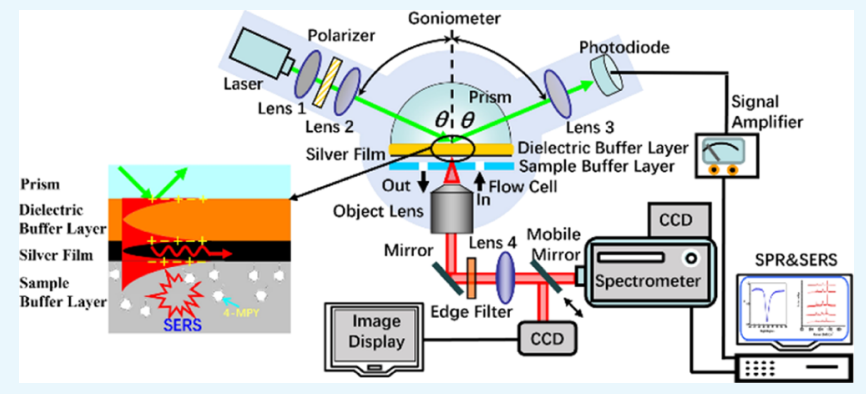
layer. The incident angle-dependent surface-enhanced Raman scattering (SERS) spectra were measured in the evanescent field. At the SPR angle, the SERS signal of the symmetric configuration was 60 times higher than that of the conventional SPR configuration. Moreover, the electric field penetration depth of the symmetric long-range surface plasmon configuration $(>1000 \mathrm{~nm})$ was longer than that of their asymmetric counterparts. The enhancement factor of the symmetric configuration was $8.6 \times 10^{7}$, which corresponded to the lowest detectable concentration for 4mercaptopyridine, reaching $1.0 \times 10^{-10} \mathrm{M}$ at the resonance angle. Thus, the symmetric LRSPR configuration has great potential for label-free sensing and detection of macromolecules and biomolecules.

\section{INTRODUCTION}

Raman spectroscopy is a key technique for the nondestructive analysis of molecular structures in biomedicine, ${ }^{1}$ materials science, ${ }^{2}$ and biochemical analysis. ${ }^{3,4}$ However, the intensity of the Raman scattered light is very weak, which limits the further development of this technique. During the past decades, many approaches have been adopted to enhance the sensitivity of Raman measurements, the most important of which is surfaceenhanced Raman scattering (SERS), which has resulted in the use of Raman spectroscopy becoming more widespread. ${ }^{5-7}$ The enhancement mechanisms of SERS can be roughly divided into two categories: electromagnetic and chemical. ${ }^{8}$ Electromagnetic enhancement is based on the amplification of an electromagnetic field near a 'hot spot' made of nanostructures. ' In recent years, with the development of nanotechnology, various nanostructured SERS substrates have been used to enhance Raman signals., ${ }^{9,10}$ However, the preparation process of these nanostructured substrates is complex and the substrates have poor uniformity. Therefore, it is necessary to develop some simple methods to prepare substrates that generate strong SERS signals. ${ }^{11}$

Surface plasmon resonance (SPR) is an important way of electromagnetic enhancement, ${ }^{12}$ which produces an extremely strong SERS signal at the resonance angle. SERS substrates based on an SPR structure comprise just one or several nanometer-thick metal or dielectric films and can be prepared simply. Various SPR structures that use the electromagnetic field enhancement of SPR excitation near the surface of a metal thin film exist. These can be classified into four types: ${ }^{13}$ conventional SPR, long-range SPR (LRSPR), ${ }^{14}$ coupled plasmon-waveguide resonance, ${ }^{15}$ and the waveguide-coupled SPR. ${ }^{16}$ The conventional SPR configuration is a Kretschmann configuration and comprises a glass prism, a thin metal film, and a sample buffer layer in sequence. The long-range SPR configuration is constructed by introducing a dielectric buffer layer between the prism and the metal layer of the conventional SPR configuration. The coupled plasmon-waveguide resonance configuration can be constructed by incorporating a waveguide layer under the metal film of the conventional SPR configuration. The waveguide-coupled SPR

Received: August 15, 2020

Accepted: December 2, 2020

Published: December 16, 2020 
is formed by sequentially adding a metal layer and a waveguide layer beneath the thin metal film of the conventional SPR configuration. At the resonance angle, these SPR structures produce strong SERS signals.

LRSPR structures include a dielectric buffer layer between the prism and the metal layer, which is the feature that distinguishes them from conventional SPR structures. ${ }^{17,18}$ The propagation length of the SPs and electromagnetic field are greater than those with conventional SPR structures. ${ }^{14} \mathrm{~A}$ symmetric LRSPR configuration can be achieved when the refractive indices of the dielectric buffer layer and the sample buffer are equal. This increases the propagation length and electromagnetic field strength near the metal surface relative to the asymmetric structures. To satisfy this symmetrical refractive index condition, a solid film with a refractive index close to that of the sample buffer is usually inserted between a Ag film and the prism; this is referred to as the dielectric buffer layer. However, the refractive index of this dielectric buffer layer is constant and cannot be changed. Thus, the symmetry condition cannot be met for measurements that involve a large change in the refractive index. At present, materials such as Cytop, $\mathrm{MgF}_{2}, \mathrm{Si}$, and $\mathrm{SiO}_{2}$ are used as the dielectric buffer. ${ }^{19}$ The refractive indices of these materials are higher than those of the liquids used in the sample buffer layer. Thus, the LRSPR configuration is not under a symmetry condition for some SERS applications. ${ }^{14,20}$ This lack of symmetry results in a shallower LRSPR dip, a weaker electromagnetic field, and even LRSPR decoupling. ${ }^{21}$ Therefore, new designed proper solutions are needed to achieve good LRSPR symmetry condition.

In this article, we propose a simple method of adjusting the refractive index of the sample buffer layer by adjusting the concentration of an ethylene glycol aqueous solution which was used as sample buffer solution in the LRSPR configuration. When the refractive indices of the sample buffer solution and dielectric buffer layer were the same, a symmetric LRSPR structure was formed. We compared the electric field strength and penetration distance and the half-peak width of the SPR curve with those of asymmetric LRSPR configuration and conventional SPR configuration. The symmetric structure had the narrowest SPR curve and highest electric field strength. Figure 1 shows the LRSPR configuration used to detect SERS spectra of 4-mercaptopyridine (4-Mpy). ${ }^{14,20}$ The LRSPR configuration is similar to that in refs. ${ }^{14,20}$ It should be noted that the LRSPs generated under the symmetric LRSPR configuration are not symmetric LRSPs. The difference between the symmetric LRSPR configuration and asymmetric

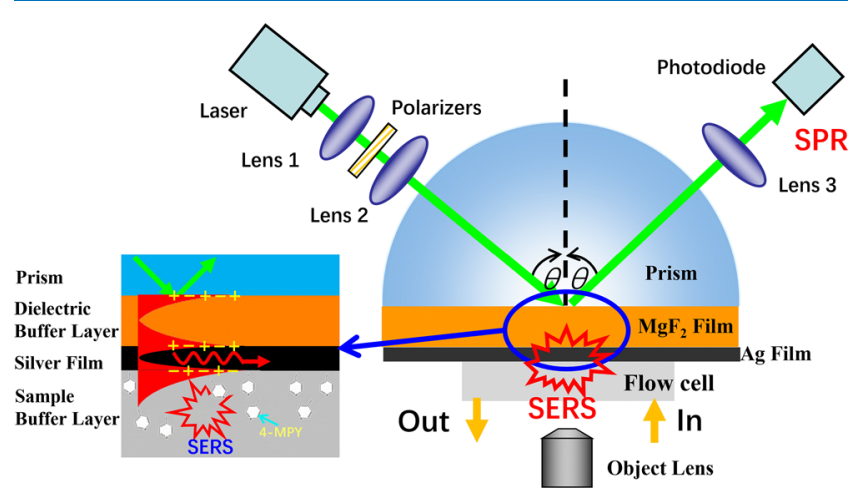

Figure 1. Setup of the LRSPR for SERS detection.
LRSPR configuration is just a difference of the degree of index matching. The intrinsic asymmetry of LRSPs is induced by the prism in the prism-coupled LRSP configuration. ${ }^{22}$

\section{THEORETICAL ANALYSIS AND NUMERICAL SIMULATION}

When a beam of laser irradiates the interface between the metal film and the prism, if the frequency and wave vector of the incident light are appropriate, the free electrons on the surface of the metal film will absorb the light energy, which will cause the oscillation of the surface charge density and produce surface plasmons. If the frequency of the incident p-polarized light matches the frequency of surface plasmons, resonant absorption can occur, which yields an extremely sharp optical reflectance spectrum. When the incident angle is the resonance angle, the intensity of the reflected light reaches the minimum value. An absorption peak is formed near the resonance angle. In the symmetric LRSPR structure, the frequency of the surface plasmon and that of the incident p-polarized light only can match at a small scanning angle, which makes the halfwidth of the LRSPR curve under the symmetrical LRSPR structure very narrow.

In the LRSPR configuration, because the metal film is wrapped between two dielectric layers with similar refractive indices, independent surface plasmon waves will be generated on both sides of the metal film and propagated along the metal/dielectric interface. When the thickness of the metal film is sufficiently thin, the surface plasmon waves generated on both sides of the metal film coupled with each other and generated long-range surface plasmons. When the refractive index of the dielectrics on both sides of the metal film is the same (symmetric LRSPR structure), the surface plasmon waves generated on the two surfaces of the metal film have the same oscillation frequency. The cross coupling between these two surface plasmon waves leads to the generation of a stronger electric field. Therefore, the electric field strength under the symmetrical LRSPR structure is the strongest.

A four-phase Kretschmann-type LRSPR configuration was used in this study. We constructed a four-phase LRSPR configuration by sequential coating of a dielectric buffer layer $\left(\mathrm{MgF}_{2}\right)$ and a metal layer (Ag) on a $\mathrm{K} 9$ prism. The LRSPR structure changed the electromagnetic field strength, the full width at half-maximum (FWHM) of the SPR curve, and the electric field penetration depth around the metallic film. We optimized the thicknesses of the $\mathrm{MgF}_{2}$ and $\mathrm{Ag}$ films based on the electric field strengths and the electric field penetration depth using the multilayer Fresnel equation. ${ }^{23}$ The simulation results show that for the symmetric LRSPR structure, for a given $\mathrm{MgF}_{2}$ film thickness, a thinner $\mathrm{Ag}$ film gives a higher electric field strength at the resonance angle. However, when the thickness of the Ag film was less than $10 \mathrm{~nm}$, the film exhibited a discontinuous island structure. Therefore, we set the Ag film thickness to $10 \mathrm{~nm}$ during the simulation.

We simulated the SPR curve and the curve of the electric field intensity as functions of incident angle for different $\mathrm{MgF}_{2}$ film thicknesses. The simulation results are shown in Figure 2. When the refractive index of the sample buffer layer was the same as that of the $\mathrm{MgF}_{2}$ film $(n=1.38)$, the electric field intensity at the resonance angle was the largest and the SPR dip was the lowest. As shown in Figure 3, the optimal $\mathrm{MgF}_{2}$ film thickness for the strongest electric field strength $\left(E^{2}\right)$ was approximately $1170 \mathrm{~nm}$ for an LRSPR configuration. 

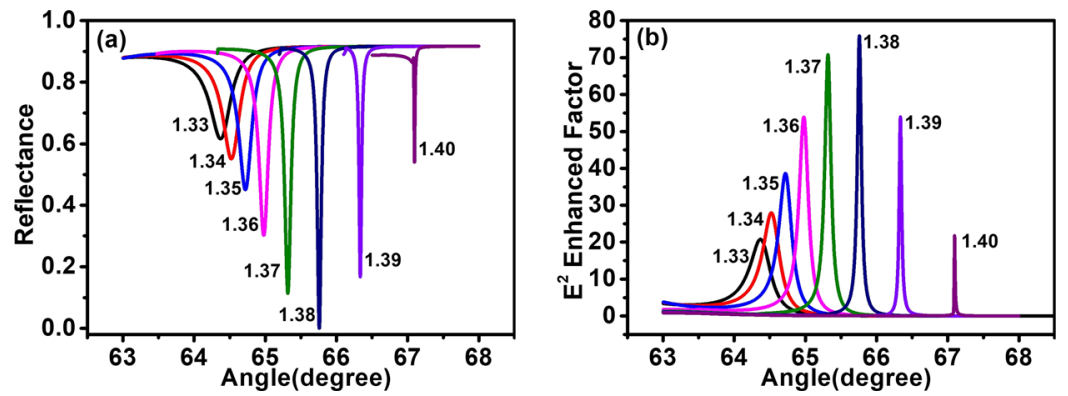

Figure 2. Theoretical calculations of (a) the effect of the refractive index of the sample buffer layer on the LRSPR curve and (b) the incident angledependent $E^{2}$ curve for a fixed $\mathrm{Ag}$ film $(n=0.13+3.19 i)$ thickness of $10 \mathrm{~nm}$ and a dielectric buffer layer $\left(\mathrm{MgF}_{2}, n=1.38\right)$ thickness of $1170 \mathrm{~nm}$. The excitation wavelength was $532 \mathrm{~nm}$.
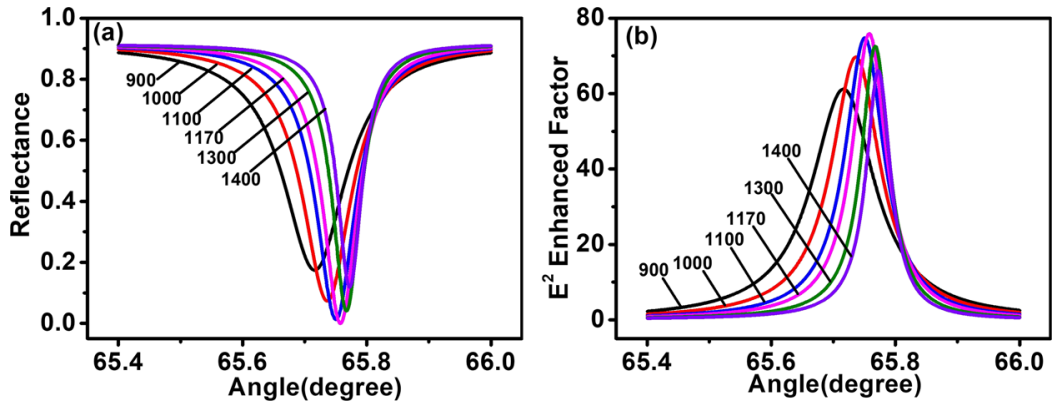

Figure 3. Theoretical calculations of (a) the effect of the dielectric buffer layer thickness on the LRSPR curve and (b) the incident angle-dependent $E^{2}$ curve for a fixed $\mathrm{Ag}$ film $(n=0.13+3.19 i)$ thickness of $10 \mathrm{~nm}$. The refractive index of the sample buffer layer was 1.38 , and the excitation wavelength was $532 \mathrm{~nm}$.

For the symmetric LRSPR configuration, the maximal electric field strength was approximately 75.88 times higher than that of the incident light, and the electric field penetration depth was $3512 \mathrm{~nm}$ for a $\mathrm{MgF}_{2}$ layer thickness of $1170 \mathrm{~nm}$. For the asymmetric LRSPR configuration, when the sample layer was water, the optimal thicknesses of the $\mathrm{MgF}_{2}$ and $\mathrm{Ag}$ films for achieving the highest electric field strength were approximately 1750 and $16 \mathrm{~nm}$, respectively, according to our published paper. ${ }^{14}$ Based on the simulated results mentioned above, a symmetric LRSPR configuration with a $\mathrm{K} 9$ glass prism $/ \mathrm{MgF}_{2}(1170 \mathrm{~nm}) / \mathrm{Ag}(10 \mathrm{~nm}) /$ ethylene glycol aqueous solution $(n=1.38)$ four-phase structure was prepared. An asymmetric LRSPR configuration with a $\mathrm{K} 9 \mathrm{prism} / \mathrm{MgF}_{2}$ $(1750 \mathrm{~nm}) / \mathrm{Ag}(16 \mathrm{~nm}) /$ water four-layer structure and a conventional SPR configuration with a $\mathrm{K} 9$ prism/ $\mathrm{Ag}(45 \mathrm{~nm}) /$ water trilayer structure were also constructed for comparison.

Figure 4 shows the simulated SPR curves, the incident angledependent $E^{2}$, the electric field distribution, and the electric field penetration depth for the conventional SPR configuration, asymmetric LRSPR configuration, and symmetric LRSPR configuration; this information is summarized in Table 1 . The simulation data used in panel $b$, panel $c$, panel e, and panel $\mathrm{f}$ are the simulation data in Figure 2 of ref 14 which were published by our group. The FWHMs of the LRSPR curve $\left(0.07^{\circ}\right)$ with symmetric configuration and the LRSPR curve $\left(0.12^{\circ}\right)$ with asymmetric configuration were much narrower than the conventional SPR curve $\left(5.21^{\circ}\right)$. The angle of incidence with the maximal electric field strength for the symmetric LRSPR configuration, asymmetric LRSPR configuration, and conventional SPR configuration was 65.75, 64.98, and $73.04^{\circ}$, respectively. The maximal electric field strength based on the symmetric LRSPR configuration was approximately 75.88 times higher than that of the incident light; this was 1.68 and 6.32 times higher than the maximal electric field strength for the asymmetric LRSPR configuration and conventional SPR configuration, respectively. The penetration depths of the evanescent field for the symmetric LRSPR configuration, asymmetric LRSPR configuration, and SPR configuration were 3512,1138, and $502 \mathrm{~nm}$, respectively. We note that the electric field enhancement and evanescent field penetration depth are strongly affected by the refractive indices of the prisms, dielectric media, and sample layers (please see the Supporting Information). The electric field strength and penetration depth of the evanescent field could be further enhanced though selection of a suitable prism and suitable dielectric layer materials.

\section{EXPERIMENTAL RESULTS AND DISCUSSION}

The film thicknesses used in the experiment are $1170 \mathrm{~nm}$ $\left(\mathrm{MgF}_{2}\right.$ film) and $10 \mathrm{~nm}$ (Ag film), because it is the best thickness according to the simulation result. After installing the prism with the $\mathrm{MgF}_{2}$ film $(1170 \mathrm{~nm})$ and $\mathrm{Ag}$ film $(10 \mathrm{~nm})$ on the SPR-SERS microspectrometer, the 4-MPY solution was injected into the flow cell and 4-MPY molecules were adsorbed on the Ag film. Then, the ethylene glycol aqueous solutions with different refractive indices were injected into the flow cell to construct different LRSPR structures. In the experiment, we sequentially injected water $(n=1.33)$, ethylene glycol aqueous solution with a refractive index of 1.38 , and ethylene glycol aqueous solution with a refractive index of 1.40 into the flow cell. We measured the SPR curve and SERS intensity curve as functions of incident angle with these three different sample buffer layers (ethylene glycol aqueous solution). The experimental results are shown in Figure 5. When the refractive index of the sample buffer layer (ethylene glycol aqueous solution) was 1.38 , the LRSPR dip was the lowest and the 


\section{Traditional SPR Configuration}
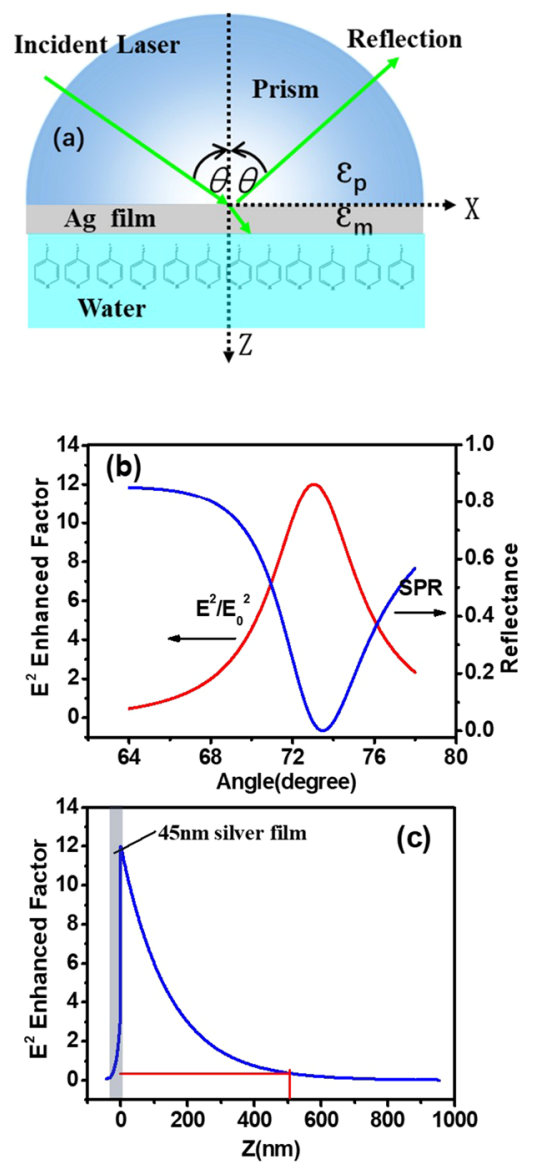

Asymmetric LRSPR

Configuration
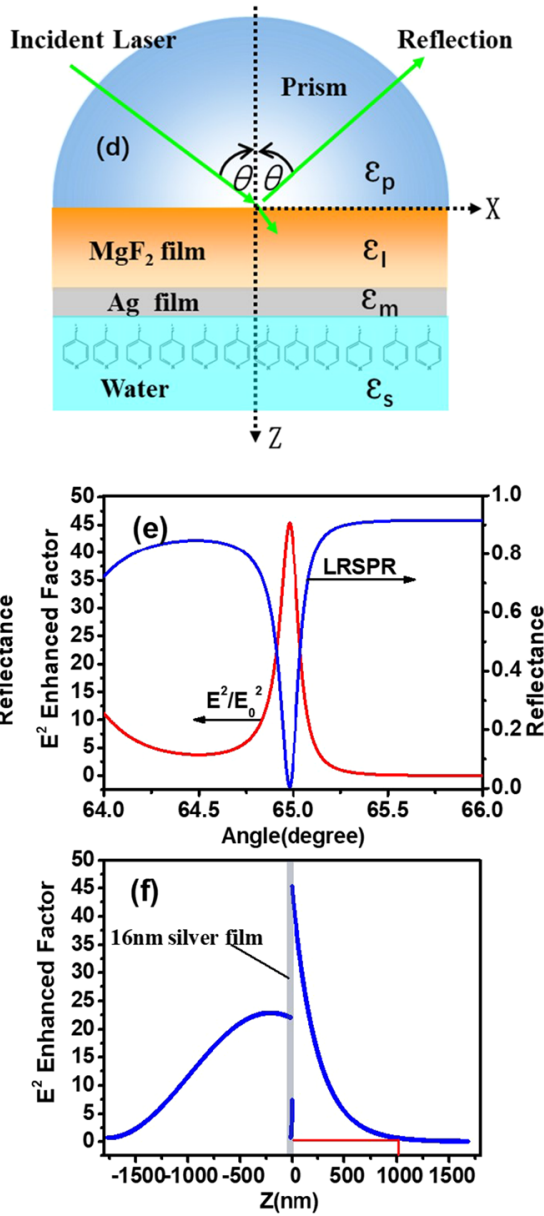

\section{Symmetric LRSPR Configuration}
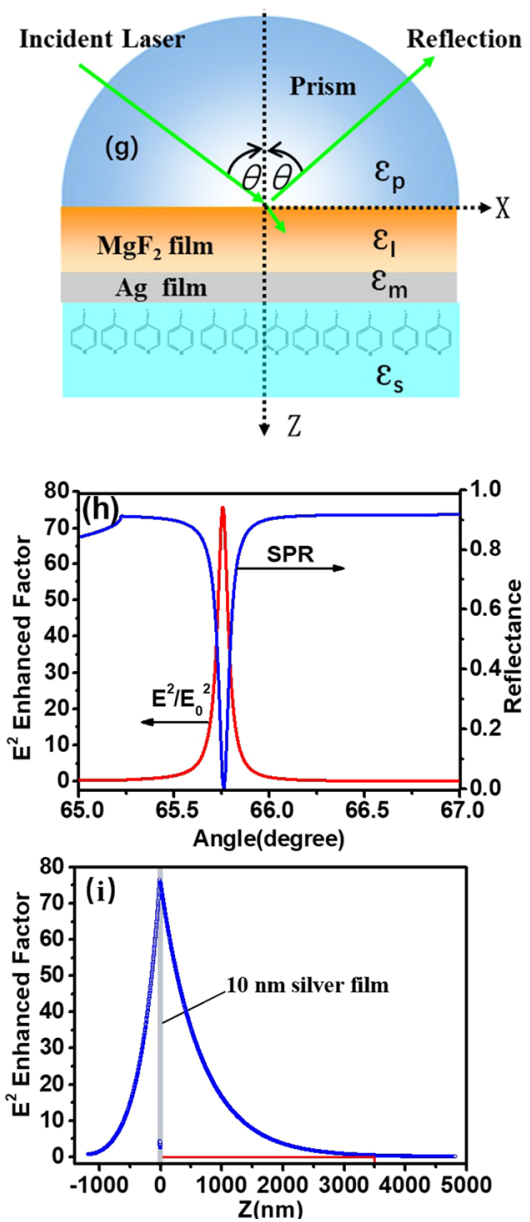

Figure 4. (a) Kretschmann prism-coupling SPR configuration. (b) Simulation of the SPR curve and angular electric field for a prism $(n=1.52$ at $532 \mathrm{~nm}$ ) $/ 45 \mathrm{~nm} \mathrm{Ag} \mathrm{film/water} \mathrm{trilayer} \mathrm{structure.} \mathrm{(c)} \mathrm{Normalized} \mathrm{electric} \mathrm{field} \mathrm{distribution} \mathrm{for} \mathrm{the} \mathrm{architecture} \mathrm{shown} \mathrm{in} \mathrm{panel} \mathrm{a.} \mathrm{(d)} \mathrm{Asymmetric}$ LRSPR configuration for LRSP excitation on the prism with a dielectric buffer layer $\left(\mathrm{MgF}_{2}, d=1750 \mathrm{~nm}\right.$ and $\left.n=1.38\right)$ and the metal layer $(\mathrm{Ag}, d=$ $16 \mathrm{~nm}$ ) followed by a sample buffer layer (water, $n=1.33$ ). (e) LRSPR curve with asymmetric configuration and angular electric field scan simulation for the architecture shown in panel d. (f) Electric field distribution for the architecture shown in panel d. (g) Symmetric LRSPR configuration for LRSP excitation on the prism with a dielectric buffer layer $\left(\mathrm{MgF}_{2}, d=1170 \mathrm{~nm}\right.$ and $\left.n=1.38\right)$ and a metal layer $(\mathrm{Ag}, d=10 \mathrm{~nm})$ followed by a sample buffer layer (ethylene glycol solution $=1.38$ ). (h) LRSPR curve and angular electric field with symmetric configuration scan simulation for the architecture shown in panel g. (i) Electric field distribution for the architecture shown in panel g. The simulation data used in panel b, panel c, panel e, and panel $\mathrm{f}$ are the simulation data in ref 14 which were published by our group.

Table 1. Comparison of the Symmetric LRSPR

Configuration, Asymmetric LRSPR Configuration, and Conventional SPR Configuration from the Simulated Data

\begin{tabular}{|c|c|c|c|c|c|}
\hline type & $\begin{array}{c}\theta \mathrm{SPR} \\
\text { (degree) }\end{array}$ & $\begin{array}{c}\text { FWHM } \\
\text { (degree) }\end{array}$ & $\begin{array}{c}\Theta \text { with } \\
E_{\max } \\
\text { (degree) }\end{array}$ & $E_{\max }^{2} / E_{\text {incident }}{ }^{2}$ & $\underset{(\mathrm{nm})}{d_{\mathrm{p}}}$ \\
\hline $\begin{array}{l}\text { conventional } \\
\text { SPR } \\
\text { configuration }^{a}\end{array}$ & 73.04 & 5.21 & 73.04 & 12.01 & 502 \\
\hline $\begin{array}{l}\text { asymmetric } \\
\text { LRSPR } \\
\text { configuration }^{b}\end{array}$ & 64.98 & 0.12 & 64.98 & 45.32 & 1138 \\
\hline $\begin{array}{l}\text { symmetric } \\
\text { LRSPR } \\
\text { configuration }^{c}\end{array}$ & 65.75 & 0.07 & 65.75 & 75.88 & 3512 \\
\hline \multicolumn{6}{|c|}{$\begin{array}{l}{ }^{a} \text { Prism } / 45 \mathrm{~nm} \mathrm{Ag} \mathrm{film} / \text { water trilayer structure. }{ }^{b} \text { Prism } / 1750 \mathrm{~nm} \\
\mathrm{MgF}_{2} / 16 \mathrm{~nm} \mathrm{Ag} \mathrm{film} / \text { water four-phase structure. }{ }^{c} \text { Prism } / 1170 \mathrm{~nm} \\
\mathrm{MgF}_{2} / 10 \mathrm{~nm} \text { Ag film/ethylene glycol aqueous solution }(n=1.38) \\
\text { four-phase structure. }\end{array}$} \\
\hline
\end{tabular}

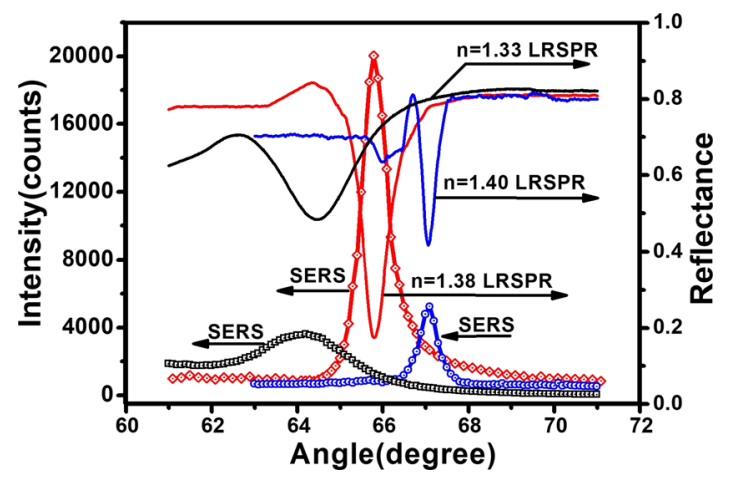

Figure 5. Comparison of angular reflectivity scans recorded using an LRSPR configuration with difference sample buffers layer (refractive indices are 1.33 (black $\square$ ), 1.38 (red $\diamond)$, and 1.40 (blue $\bigcirc)$ ).

intensity of SERS was the largest. This result demonstrates that the symmetrical LRSPR structure has the strongest ability to 

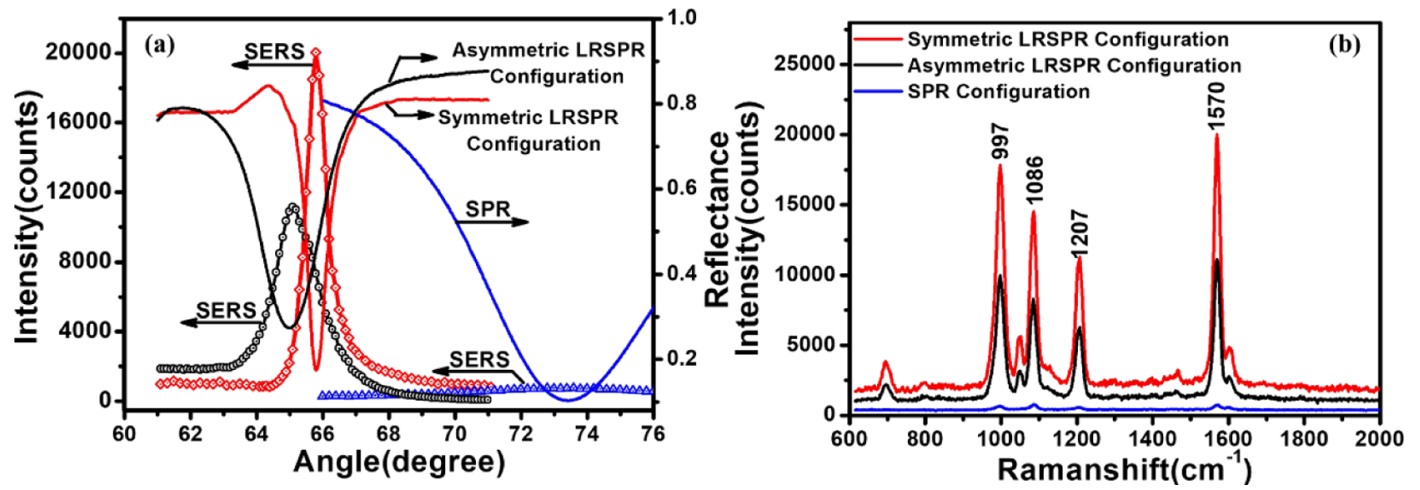

Figure 6. (a) Comparison of angular reflectivity scans recorded using the normal SPR configuration, asymmetric LRSPR configuration, and symmetric LRSPR configurations. The angle-dependent SP field-enhanced SERS intensities from the SPR (blue $\Delta$ ), asymmetric LRSPR (black O), and symmetric LRSPR configurations (red $\diamond$ ) are also shown. (b) SERS spectra excited using the symmetric LRSPR configuration at a resonance angle of $65.80^{\circ}$ (upper curve), SERS spectra excited using the asymmetric LRSPR configuration at a resonance angle of $65.00^{\circ}$ (middle curve), and SPs excited with the normal SPR configuration at a resonance angle of $73.6^{\circ}$ (bottom curve).

\section{Asymmetric LRSPR Configuration}

\section{Symmetric LRSPR Configuration}
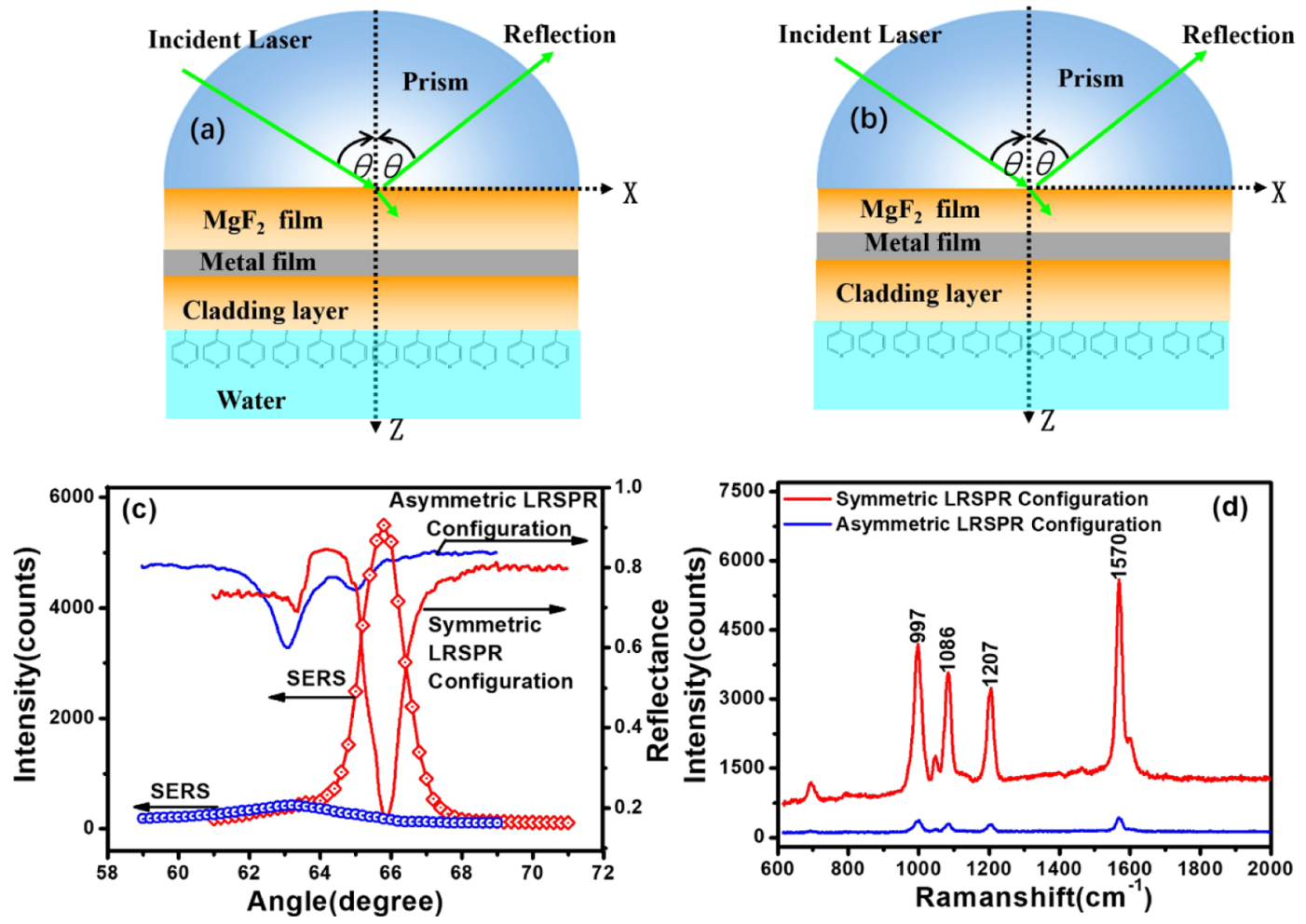

Figure 7. (a) Asymmetric LRSPR configuration for LRSP excitation with a $1000 \mathrm{~nm}$ cladding layer. (b) Configuration for LRSP excitation using the symmetric LRSPR configuration with a $1000 \mathrm{~nm}$ cladding layer. (c) Comparison of angular reflectivity scans recorded using the configurations in $(\mathrm{a}, \mathrm{b})$, along with plots of the SERS intensities $\left(1574 \mathrm{~cm}^{-1}\right)$ of the SP field-enhanced SERS for the asymmetric configuration and symmetric LRSPR configuration. (d) SERS spectra excited by SPs using the asymmetric LRSPR configuration at $63.20^{\circ}$ and the symmetric LRSPR configuration at $65.80^{\circ}$.

enhance electric field strength, which is consistent with the simulation results.

We also compared the electric field strengths, FWHM of the SPR curve, and electric field penetration depth for the conventional SPR configuration, asymmetric LRSPR configuration, and symmetric LRSPR configuration. For the SERS measurements, the Raman probe molecule, 4-Mpy, was assembled on the bare Ag surfaces of the three configurations. The SPR curves and incident angle-dependent SERS spectra of 4-Mpy were measured simultaneously using an SPR-SERS microspectrometer (see the Experimental Methods). Figure 6a shows the reflectivity curves and incident angle-dependent SERS intensity $\left(1570 \mathrm{~cm}^{-1}\right)$ curves for the different configurations. The highest SERS signal intensities appeared in the vicinity of the resonance angles for all the three cases. Figure $6 \mathrm{~b}$ compares the SERS spectra obtained using the three configurations near the SPR angles $\left(65.80^{\circ}\right.$ for the symmetric LRSPR configuration, $65.00^{\circ}$ for the asymmetric LRSPR configuration, and $73.60^{\circ}$ for the conventional SPR configuration). The SERS signal $\left(1570 \mathrm{~cm}^{-1}\right)$ obtained from the 
symmetric LRSPR configuration was approximately 2 and 40 times higher than that from the asymmetric LRSPR configuration and conventional SPR configurations, respectively. We attribute this SERS enhancement to the higher electric field strengths provided by the LRSPs than by conventional SPs.

The experimentally observed SPR angles (Figure 6a) agreed well with the simulated results (Figure $4 \mathrm{~b}, \mathrm{e}, \mathrm{h}$ ). A narrower FWHM of the SPR curve corresponded to a higher electric field strength and greater electric field penetration depth, which is conducive for SERS excitation and signal detection.

In our setup, the SPR and SERS collection systems lie on different sides of the prism. This allows SPR and SERS detection to be achieved simultaneously, which avoids SERS signal losses. In addition, the excitation and detection of SERS from the evanescent field are important because the baseline of the obtained spectra was suppressed and a high signal-to-noise ratio was achieved.

The LRSPR configuration gives a greater electric field penetration depth, which can be used to excite a higher number of probe molecules during SERS detection and thus increase the SERS signal intensity. To demonstrate this effect, a cladding layer $\left(\mathrm{MgF}_{2}, d=1000 \mathrm{~nm}\right)$ was constructed on the $\mathrm{Ag}$ film for both the asymmetric configuration and symmetric LRSPR configuration (Figure 6a,b). The cladding layer kept the analytes $1000 \mathrm{~nm}$ from the $\mathrm{Ag}$ film. The experimental data in Figure 7 are obtained under the symmetric LRSPR configuration and asymmetric LRSPR configuration after adding a cladding layer. Figure $7 \mathrm{c}$ shows the reflectivity curves and the incident angle-dependent SERS intensities (1570 $\mathrm{cm}^{-1}$ ) obtained using the configurations in Figure 7a,b. The resonance angles of the asymmetric configuration and symmetric configuration were 63.12 and $65.88^{\circ}$, respectively. Figure $7 \mathrm{~d}$ compares the SERS spectra obtained in the vicinity of the resonance angles for the two configurations. A highquality SERS spectrum was obtained using the symmetric configuration, whereas a low-quality SERS signal was recorded using the asymmetric configuration. The evanescent field penetration depth of the symmetric LRSP configuration was at least $1000 \mathrm{~nm}$, and that of the asymmetric LRSP configuration was about $1000 \mathrm{~nm}$. On the one hand, longer penetration depths can not only excite a higher number of probe molecules but are also beneficial in coupling with other surface plasmon modes. Both of these effects serve to enhance the SERS signal. On the other hand, longer evanescent field penetration depth is beneficial to expand the applications of SERS to multilayer systems, such as cell detection. The third, longer evanescent field penetration depth makes it possible to use Ag with a stronger electric field enhancement capability instead of an inert noble metal such as $\mathrm{Au}$ in the experiment without worrying about the oxidation of $\mathrm{Ag}$.

The enhancement factor of 4-Mpy was calculated using the symmetric LRSPR configuration. We compared the fieldenhanced SERS with symmetric LRSPR configuration at 999 $\mathrm{cm}^{-1}$ with the Raman signal at $992 \mathrm{~cm}^{-1}$. The enhancement factor was estimated to be $8.6 \times 10^{7}$. Details of the enhancement factor estimation can be found in the Supporting Information. Figure 8 shows that SERS spectra of 4-Mpy with the concentration range from $1.0 \times 10^{-6}$ to $1.0 \times 10^{-10} \mathrm{M}$. A limit of detection of $1.0 \times 10^{-10} \mathrm{M}$ was achieved.

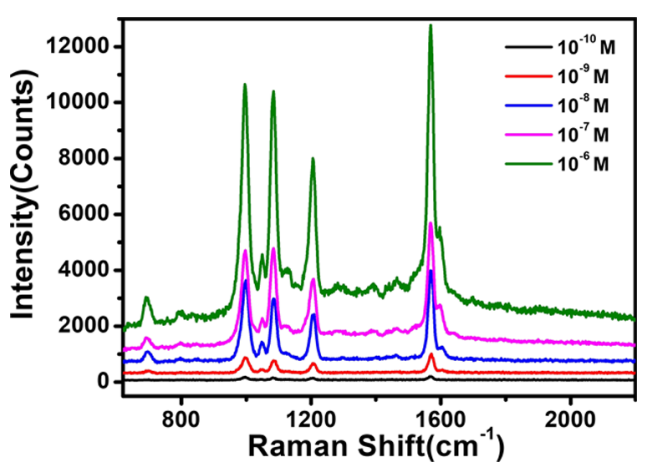

Figure 8. Concentration-dependent SERS spectra of 4-Mpy excited using the symmetric LRSPR configuration at the resonance angle.

\section{CONCLUSIONS}

We present a symmetric LRSPR configuration to enhance Raman scattering. We experimentally showed that the symmetric LRSPR configuration results in narrower angular resonance curves, longer penetration depths, and stronger electric fields than those for an asymmetric LRSPR configuration and conventional SPR configuration. Numerical simulations helped explain these observations. The symmetric LRSPR structure constructed using an ethylene glycol aqueous solution was confirmed to enhance the evanescent field penetration depth and the local electric field on the Ag surface that interacted with the samples. The symmetric LRSPR configuration process has a number of important advantages for Raman enhancement. First, the strong electric field at the metal interface is useful for SERS signal excitation. Moreover, the narrower angular distribution of the electric field is beneficial for SERS signal detection. In addition, the greater penetration depth leads to the excitation of a greater number of probe molecules, which is important for expanding the applications of SERS to multilayer systems, such as cell detection. The SERS enhancement factor with the symmetric LRSPR configuration was as high as $8.6 \times 10^{7}$. Thus, our plasmonic configuration has broad application prospects in plasmonic devices based on high-efficiency light harvesting and radiation.

\section{EXPERIMENTAL METHODS}

The SPR and LRSPR curves and the incident angle-dependent SERS spectra were measured simultaneously using an inhouse-built, angle-dependent SPR-SERS microspectrometer that can be found in the Supporting Information.

We constructed conventional SPR configuration, asymmetric LRSPR configuration, and symmetric LRSPR configuration. All three configurations were Kretschmann type. $\mathrm{MgF}_{2}(n=1.38$ at $532 \mathrm{~nm})$ and $\mathrm{Ag}(n=0.13+3.19 i$ at $532 \mathrm{~nm})$ films with various thicknesses were deposited on the bottom of $\mathrm{K} 9$ prisms $(n=1.52$ at $532 \mathrm{~nm})$ by vacuum evaporation at a pressure of $8.0 \times 10^{-4} \mathrm{~Pa}$. The deposited film thicknesses were measured using a surface profiler (Dektak 150, Veeco).

For the Raman measurements, a 4-Mpy solution $\left(10^{-5} \mathrm{M}\right)$ was injected into the flow cell for $1 \mathrm{~h}$. 4-Mpy molecules were adsorbed on the $\mathrm{Ag}$ film by mercapto groups to form an assembled monolayer. Water was then injected into the flow cell, and the 4-Mpy molecules that were not adsorbed on the $\mathrm{Ag}$ film were washed away. Finally, ethylene glycol aqueous solutions with different refractive indices were sequentially injected into the flow cell to form different LRSPR 
configurations. The 4-Mpy monolayer acts as the signal emitter in our design; it is excited by the SPs and radiated SERS signals in the evanescent field. We used an SPR-SERS microspectrometer to record the SPR curves and incident angledependent SERS spectra of 4-Mpy simultaneously.

\section{ASSOCIATED CONTENT}

\section{SI Supporting Information}

The Supporting Information is available free of charge at https://pubs.acs.org/doi/10.1021/acsomega.0c03923.

Simulation of the electronic field and SPR curve with the incidence angle, calculation for the SERS enhancement factor of 4-Mpy, and angle-dependent SPR-SERS microspectrometer (PDF)

\section{AUTHOR INFORMATION}

\section{Corresponding Author}

Liping Wang - State Key Laboratory of Applied Optics, Changchun Institute of Optics, Fine Mechanics and Physics, Chinese Academy of Sciences, Changchun 130033, China; University of Chinese Academy of Sciences, Beijing 100049, China; orcid.org/0000-0002-2200-4032;

Email: wlp8121@126.com

\section{Authors}

Yu Liu - State Key Laboratory of Applied Optics, Changchun Institute of Optics, Fine Mechanics and Physics, Chinese Academy of Sciences, Changchun 130033, China; University of Chinese Academy of Sciences, Beijing 100049, China; (1) orcid.org/0000-0002-9815-0060

Haitao Zhang - State Key Laboratory of Applied Optics, Changchun Institute of Optics, Fine Mechanics and Physics and Key Laboratory of Microelectronics Devices \& Integrated Technology, Institute of Microelectronics, Chinese Academy of Sciences, Changchun 130033, China; School of Microelectronics, University of Chinese Academy of Sciences, Beijing 100049, China

Yijia Geng - State Key Laboratory of Supramolecular Structure and Materials, Jilin University, Changchun 130012, China

Shuping Xu - State Key Laboratory of Supramolecular Structure and Materials, Jilin University, Changchun 130012, China; orcid.org/0000-0002-6216-6175

Weiqing Xu - State Key Laboratory of Supramolecular Structure and Materials, Jilin University, Changchun 130012, China; o orcid.org/0000-0002-1947-317X

Jie Yu - State Key Laboratory of Applied Optics, Changchun Institute of Optics, Fine Mechanics and Physics, Chinese Academy of Sciences, Changchun 130033, China

Wenyuan Deng - State Key Laboratory of Applied Optics, Changchun Institute of Optics, Fine Mechanics and Physics, Chinese Academy of Sciences, Changchun 130033, China

Bo Yu - State Key Laboratory of Applied Optics, Changchun Institute of Optics, Fine Mechanics and Physics, Chinese Academy of Sciences, Changchun 130033, China

Complete contact information is available at:

https://pubs.acs.org/10.1021/acsomega.0c03923

\section{Funding}

National Natural Science Foundation of China (nos. 21603211, 61178020, 61505202, and 61605201); Jilin Province Young Talent Fund Projects (no. 20180520156JH);
Science and Technology Development Project of Jilin Province (no. 20180101284JC); Scientific Instrument Developing Project of the Chinese Academy of Sciences (no. D02020006177); Strategic Priority Research Program of Chinese Academy of Sciences (no. XDA22020502).

\section{Notes}

The authors declare no competing financial interest.

\section{ACKNOWLEDGMENTS}

The authors thank the support of the ShuGuang Project of CIOMP and the Autonomous Fund of SKLAO.

\section{REFERENCES}

(1) Qian, X.; Peng, X.-H.; Ansari, D. O.; Yin-Goen, Q.; Chen, G. Z.; Shin, D. M.; Yang, L.; Young, A. N.; Wang, M. D.; Nie, S. In vivo tumor targeting and spectroscopic detection with surface-enhanced Raman nanoparticle tags. Nat. Biotechnol. 2008, 26, 83-90.

(2) Zou, H.; Ren, G.; Shang, M.; Wang, W. One-step, seedless, fabrication of three-dimensional gold meso-flowers (3D-AuMFs) with high activities in catalysis and surface-enhanced Raman scattering. Mater. Chem. Phys. 2016, 176, 115-120.

(3) Zeiri, L.; Bronk, B. V.; Shabtai, Y.; Eichler, J.; Efrima, S. Surfaceenhanced Raman spectroscopy as a tool for probing specific biochemicalcomponentsinbacteria. Appl. Spectrosc. 2004, 58, 33-40.

(4) Culha, M. Surface-enhanced Raman scattering: an emerging label-free detection and identification technique for proteins. Appl. Spectrosc. 2013, 67, 355-364.

(5) Ivanov, V. G.; Vlakhov, E. S.; Stan, G. E.; Zamfirescu, M.; Albu, C.; Mihailescu, N.; Negut, I.; Luculescu, C.; Socol, M.; Ristoscu, C.; Mihailescu, I. N. Surface-enhanced Raman scattering activity of niobium surface after irradiation with femtosecond laser pulses. $J$. Appl. Phys. 2015, 118, 203104.

(6) Zhang, R.; Zhang, Y.; Dong, Z. C.; Jiang, S.; Zhang, C.; Chen, L. G.; Zhang, L.; Liao, Y.; Aizpurua, J.; Luo, Y.; Yang, J. L.; Hou, J. G. Chemical mapping of a single molecule by plasmon-enhanced Raman scattering. Nature 2013, 498, 82-86.

(7) Liu, Y.; Xu, S.; Li, H.; Jian, X.; Xu, W. Localized and Propagating Surface Plasmon Co-enhanced Raman Spectroscopy Based on Evanescent Field Excitation. Chem. Commun. 2011, 47, 3784-3786.

(8) Ding, S.-Y.; You, E.-M.; Tian, Z.-Q.; Moskovits, M. Electromagnetic theories of surface-enhanced Raman spectroscopy. Chem. Soc. Rev. 2017, 46, 4042-4076.

(9) Ding, S.-Y.; Yi, J.; Li, J. F.; Ren, B.; Wu, D. Y.; Panneerselvam, R; Tian, Z. Q. Nanostructure-based plasmon-enhanced Raman spectroscopy for surface analysis of materials. Nat. Rev. Mater. 2016, $1,16036$.

(10) Li, J. F.; Huang, Y. F.; Ding, Y.; Yang, Z. L.; Li, S. B.; Zhou, X. S.; Fan, F. R.; Zhang, W.; Zhou, Z. Y.; Wu, D. Y.; et al. Shell-isolated nanoparticle-enhanced Raman spectroscopy. Nature 2010, 464, 392395.

(11) Wang, Z.; Zervas, M. N.; Bartlett, P. N.; Wilkinson, J. S. Surface and waveguide collection of Raman emission in waveguide-enhanced Raman spectroscopy. Opt. Lett. 2016, 41, 4146-4149.

(12) Liu, Y.; Xu, S.; Tang, B.; Wang, Y.; Zhou, J.; Zheng, X.; Zhao, B.; Xu, W. Simultaneous Measurement of Surface Plasmon Resonance and Surface-Enhanced Raman Scattering. Rev. Sci. Instrum. 2010, 81, 036105-036107.

(13) Chien, F.-C.; Chen, S.-J. A sensitivity comparison of optical biosensors based on four different surface plasmon resonance modes. Biosens. Bioelectron. 2004, 20, 633-642.

(14) Liu, Y.; Xu, S.; Xuyang, X.; Zhao, B.; Xu, W. Long-Range Surface Plasmon Field-Enhanced Raman Scattering Spectroscopy Based on Evanescent Field Excitation. J. Phys. Chem. Lett. 2011, 2, 2218-2222.

(15) Tian, Y.; Wang, H.; Xu, W.; Liu, Y.; Xu, S. Waveguide-coupled localized surface plasmon resonance for surface-enhanced Raman 
scattering: Antenna array as emitters. Sens. Actuators, B 2019, 280, $144-150$.

(16) Zhang, H.; Geng, Y.; Xu, S.; Xu, W.; Tian, Y.; Yu, J.; Deng, W.; Yu, B.; Yu Liu, Y. Surface Plasmon Field-Enhanced Raman Scattering Based on Evanescent Field Excitation of Waveguide-Coupled Surface Plasmon Resonance Configuration. J. Phys. Chem. C 2020, 124, $1640-1645$.

(17) Sarid, D. Long-range surface plasma waves on very thin metal films. Phys. Rev. Lett. 1981, 47, 1927-1930.

(18) Nenninger, G. G.; Tobiška, P.; Homola, J.; Yee, S. S. Longrange surface plasmons for high-resolution surface plasmon resonance sensors. Sens. Actuators, B 2001, 74, 145-151.

(19) Jing, J.-Y.; Wang, Q.; Zhao, W.-M.; Wang, B.-T. Long-range surface plasmon resonance and its sensing applications: A review. Optic Laser. Eng. 2019, 112, 103-118.

(20) Xuan, X.; Xu, S.; Liu, Y.; Li, H.; Xu, W.; Lombardi, J. R. A Long-Range Surface Plasmon Resonance/Probe/Silver Nanoparticle (LRSPR-P-NP) Nanoantenna Configuration for Surface-Enhanced Raman Scattering. J. Phys. Chem. Lett. 2012, 3, 2773-2778.

(21) Kan, T.; Kojo, H.; Iwase, E.; Matsumoto, K.; Shimoyama, I. Long-range surface plasmon resonance sensor with liquid microchannels to maintain the symmetry condition of the refractive index. $J$. Micromech. Microeng. 2010, 20, 125005.

(22) WENDLER, L.; HAUPT, R. An Improved Virtual Mode Theory of ATR Experiments on Surface Polaritons. Phys. Status Solidi B 1987, 143, 131-148.

(23) Ekgasit, S.; Thammacharoen, C.; Knoll, W. Surface Plasmon Resonance Spectroscopy Based on Evanescent Field Treatment. Anal. Chem. 2004, 76, 561-568. 